\title{
El Perú en las Naciones Unidas: el desarrollo sostenible y el sostenimiento de la paz
}

\author{
Gustavo Meza-Cuadra*
}

\begin{abstract}
RESUMEN
Paz en las Naciones Unidas, a pesar de las críticas sobre su eficacia y la revisión de los compromisos multilaterales de Estados Unidos, la ONU se está renovando a través de dos nuevos marcos ambiciosos y orientados a la acción: la Agenda 2030, que compromete a los países a transformar el mundo alcanzando los Objetivos de Desarrollo Sostenible (ODS), y el enfoque complementario del objetivo general de sostener la paz, destacado por el nuevo secretario general, António Guterres, para abordar las causas profundas de los conflictos y favorecer la diplomacia preventiva. El Perú, sobre la base de su tradicional compromiso con el multilateralismo, ha desempeñado un papel importante en el establecimiento de ambos marcos y está comprometido con su implementación. Por un lado, al alinear sus políticas y estrategias nacionales de desarrollo con los ODS. Por otra parte, al hacer de la paz sostenible una orientación básica para su participación en el Consejo de Seguridad como miembro no permanente durante el bienio 2018-2019.
\end{abstract}

Palabras clave: Agenda 2030, desarrollo sostenible, paz.

\section{Peru in the United Nations: Sustainable Development and Peace Sustaining}

\section{Abstract}

Peace The UN, despite spread criticism about its efficacy and the revision of US multilateral engagements, is renewing itself through two new ambitious and action oriented frameworks: the 2030 Agenda, which commits countries to transform the world by achieving the Sustainable Development Goals (SDGs), and the complementary approach to the overarching goal of sustaining peace emphasized by the new Secretary General, António Guterres, in order to address the root causes of conflicts and favour preventive diplomacy. Peru, building on its traditional commitment to multilateralism, has played an important role in setting up

* Máster en Política Internacional Pública de la Universidad de Johns Hopkins en Washington, DC; máster en Diplomacia y Gestión de Organizaciones Internacionales, de la Universidad de París. Embajador del Servicio Diplomático del Perú, es actualmente representante permanente del Perú ante las Naciones Unidas. Correo electrónico: GMezaCuadra@unperu.org 
both frameworks and is committed to their implementation. On the one hand, by aligning its national developmental policies and strategies with the SDGs. On the other, by making sustaining peace a core guideline for its participation in the Security Council as a nonpermanent member during the biennium 2018-2019.

Keywords: 2030 Agenda, Sustainable Development, Peace. 


\section{Introduccción}

El presidente del Perú, Pedro Pablo Kuczynski, fue en febrero pasado uno de los primeros jefes de estado en visitar, en la sede principal de las Naciones Unidas en Nueva York, a su nuevo secretario general, el portugués António Guterres, entonces recién inaugurado en el cargo. Ambos líderes sostuvieron un diálogo abierto y cordial sobre diversos asuntos de la agenda internacional, en el que el mandatario peruano reafirmó el compromiso del Perú con el multilateralismo y con la Organización, enfatizando la necesidad de fortalecerlos para enfrentar desafíos globales como la pobreza, el cambio climático y las crisis que amenazan la paz y la seguridad internacionales.

El mensaje transmitido tiene como referente la coyuntura de incertidumbre sobre el futuro del orden internacional vigente, debido, entre otros desafíos, al surgimiento electoral de los nacionalismos en varias democracias importantes. Su relevancia es consistente con la historia de uno de los 51 países firmantes de la Carta de San Francisco con la que en 1945 se creó la ONU. En sus siete décadas de existencia, la diplomacia peruana ha participado activamente y ha hecho contribuciones importantes en sus tres pilares: paz y seguridad internacionales, desarrollo y derechos humanos.

En efecto, el Perú cumplió un destacado papel en la solución de graves conflictos durante el período de la Guerra Fría. Fue pionero en el desarrollo del derecho del mar. Asumió liderazgos en el proceso de descolonización y en la búsqueda de un nuevo orden económico internacional. Numerosos instrumentos que conforman la llamada maquinaria del desarme, son producto de las excepcionales calidades de diversos juristas y diplomáticos peruanos. Peruanos ilustres como Víctor Andrés Belaúnde, José Luis Bustamante y Rivero, y Javier Pérez de Cuéllar, han ejercido a cabalidad los más altos cargos de la Organización. Todo lo cual refleja la vocación multilateral del Estado peruano, cuya Cancillería muy tempranamente comprendió que una de las líneas maestras de la política exterior de un país pacífico y abierto al mundo debía ser el desarrollo de una activa diplomacia orientada a la construcción de un entorno y un sistema internacional favorables a su seguridad y desarrollo, sobre la base del derecho internacional, la prevención de conflictos, la solidaridad y la cooperación.

Es mucho lo que se ha hecho en ese sentido. No obstante, en los últimos años, se ha incrementado una percepción sobre la incapacidad o la parálisis de la ONU y de su Consejo de Seguridad, debida a las atroces situaciones observadas en Siria, en el Medio Oriente en general, y en determinados países del África, que han provocado un flujo masivo de víctimas fatales, de migrantes y de desplazados'. Incluso en el ámbito del

\footnotetext{
1 El último informe sobre Tendencias Globales publicado en 2016 por la Oficina del Alto Comisionado de las Naciones Unidas para los Refugiados (UNHCR), arroja la cifra récord de 65,3 millones de personas, o una de cada
} 
desarrollo, donde gracias a los Objetivos de Desarrollo del Milenio se ha registrado una importante reducción de la pobreza en el mundo, es frecuente la percepción de la Organización como un ente excesivamente burocratizado, en el cual la proliferación de agencias, fondos y programas desarticulados, no se ajusta a las urgentes necesidades del mundo en desarrollo. Máxime cuando se afronta el desafío adicional de competir con la creciente demanda de recursos de emergencia para atender las crisis humanitarias.

Entre las limitaciones para una acción más efectiva de la Organización, se encuentran de un lado las diferencias y la vigencia del derecho de veto de los miembros permanentes del Consejo de Seguridad, y de otro una alta fragmentación de la gobernanza de la economía mundial y del desarrollo. La gobernanza económica compete principalmente a las instituciones de Bretton Woods, el Fondo Monetario Internacional y el Banco Mundial, aunque las decisiones más importantes sean tomadas en los ámbitos del G20 y del G7. En comparación, el Consejo Económico y Social de la ONU (ECOSOC) ha cumplido a lo largo de su historia un papel menor y de alcance limitado. Conformado por 54 estados elegidos por períodos de tres ańos, las funciones principales del ECOSOC pasan por coordinar al sistema de las Naciones Unidas para el desarrollo y preparar propuestas favorables al desarrollo sostenible a través de sus comisiones regionales y otros órganos subsidiarios que no siempre cuentan con los recursos adecuados para ello. La Organización para la Cooperación y el Desarrollo Económico (OCDE) responde en parte al interés de los países desarrollados por contar con un organismo más eficiente y eficaz para conducir la cooperación internacional para el desarrollo económico.

La persistencia de crisis humanitarias, de la pobreza y el hambre, y el incremento de la vulnerabilidad humana, ponen en cuestión la efectividad de la ONU -única organización representativa de la comunidad internacional en su conjunto- para cumplir con los fines y propósitos consagrados en su Carta. Sin embargo, hay dos recientes desarrollos que podrían representar un punto de inflexión en el papel que ha venido cumpliendo la Organización, tanto en el ámbito del desarrollo como en el de la paz y la seguridad internacionales: la Agenda 2030 para el Desarrollo Sostenible, y la agenda del sostenimiento de la paz.

Ambas agendas, sumadas al Acuerdo de París sobre el Cambio Climático, la Agenda de Addis Abeba sobre Financiación para el Desarrollo, y el Marco de Sendai sobre Prevención de Riesgo de Desastres, ofrecen marcos de acción favorables a una renovación de la ONU, y en concreto plantean nuevos paradigmas para respuestas más coherentes e integradas por parte de la comunidad internacional a los desafíos que esta enfrenta. Al momentum suscitado por su adopción se suma la elección de un

113 personas, desplazadas de sus hogares debido a conflictos o persecuciones en el mundo en 2015. 
nuevo secretario general, António Guterres, de gran experiencia y prestigio, que ha destacado entre sus prioridades la de garantizar una oportuna prevención de los conflictos a partir de una visión integral del desarrollo sostenible y el sostenimiento de la paz como objetivos que se refuerzan mutuamente.

Los comentarios que siguen pasan revista a las implicancias y potencialidades de estos dos desarrollos desde la perspectiva peruana, considerando que las más altas autoridades del gobierno han señalado la plena coincidencia de los planes nacionales de desarrollo con la Agenda 2030², y que en los lineamientos de acción que el Perú ha dado a conocer en el contexto de su incorporación al Consejo de Seguridad durante el período 2018-2019 se prioriza la prevención de los conflictos y el sostenimiento de la paz. Tienen en cuenta además el complejo contexto internacional que enfrenta la ONU y su nuevo secretario general.

\section{El Perú y la Agenda 2030 para el Desarrollo Sostenible}

Por primera vez en la historia de la humanidad, la comunidad internacional cuenta con una ambiciosa agenda de alcance universal y de vocación transformadora, cuyo nivel de ambición pasa por erradicar la pobreza en todas sus formas y dimensiones y asegurar el desarrollo sostenible en sus tres dimensiones - económica, social y ambiental- Ello en un plazo de quince años. Es de destacar que la adopción de la Agenda 2030 y los Objetivos de Desarrollo Sostenible (ODS) por 193 países en setiembre de 2015 fue posible gracias a un amplio consenso, registrado en un proceso de carácter inclusivo y participativo, en torno a la necesidad de enfrentar juntos tales desafíos comunes, construyendo sobre el progreso registrado y la metodología avanzada por los Objetivos de Desarrollo del Milenio (ODM).

Es de recordar que, en setiembre de 2000, 189 países adoptaron la Declaración del Milenio, que estableció el objetivo principal de luchar contra la pobreza y la necesidad de fijar objetivos claros y un plazo para ello. El año siguiente un grupo de expertos presentó los ocho ODM y sus veintiún metas, que deberían ser alcanzadas en el año 2015 y que han tenido un impacto muy positivo, habiendo movilizado y favorecido la convergencia de todos los actores comprometidos con el desarrollo a nivel mundial: las agencias, fondos y programas de las Naciones Unidas, las insti-

\footnotetext{
2 Mensaje a la Nación del Presidente de la República Pedro Pablo Kuczynski, 28 de julio de 2016; Intervención del Presidente de la República Pedro Pablo Kuczynski en el Debate de Apertura del $71^{\circ}$ período de sesiones de la Asamblea General de las Naciones Unidas, 20 de setiembre de 2016; Presentación del Presidente del Consejo de Ministros y su Gabinete ante el Congreso de la República, 18 de agosto de 2016; Palabras del Ministro de Relaciones Exteriores, Embajador Ricardo Luna, con ocasión del $71^{\circ}$ Aniversario de la Organización de las Naciones Unidas, 24 de octubre de 2016.
} 
tuciones financieras internacionales, las diversas fuentes cooperantes, incluyendo al Comité de Asistencia al Desarrollo de la OCDE, y los propios gobiernos del mundo en desarrollo.

Muchos países cumplieron con la mayoría de los objetivos trazados —entre ellos, en forma adelantada, el Perú- y la comunidad internacional pudo comprobar la efectividad de orientar todos los esfuerzos hacia el logro de objetivos comunes. Sin perjuicio de ello, de cara a la actual Agenda 2030, también ha sido importante reconocer las limitaciones de los ODM. Se suelen destacar tres: (i) al no haber participado en su elaboración, los Estados tomaron mucho tiempo en hacerlos suyos; (ii) no se estableció desde un inicio un mecanismo de monitoreo de la implementación por parte de los Estados; y (iii) tuvieron un enfoque muy centrado en los países más pobres y en la relación cooperante/receptor o norte/sur.

A ello es posible añadir una suerte de pecado original, derivado de la premura y falta de transparencia en su elaboración. Uno de los inspiradores de los ODM, Mark Malloch-Brown, entonces administrador del Programa de las Naciones Unidas para el Desarrollo, refirió hace algunos años que, cuando el documento con los objetivos ya se había enviado a la imprenta, al cruzarse en un corredor de Naciones Unidas con su colega del Programa de las Naciones Unidas para el Medio Ambiente, se percató de que habían omitido un objetivo sobre sostenibilidad. Fue así que se agregó el objetivo siete: Asegurar la sostenibilidad ambiental (The Guardian, 2012).

Para el año 2012, la persistencia de la pobreza y su interdependencia con otros problemas y desafíos globales hicieron evidente la necesidad de contar con una agenda más ambiciosa, que integre las tres dimensiones del desarrollo sostenible económica, social y ambiental - para poder erradicar definitivamente la pobreza en todas sus formas y en todo lugar. Así, en la Conferencia Mundial sobre Desarrollo Sostenible Río + 20, la comunidad internacional decidió construir sobre la base de la experiencia ganada con los ODM, e iniciar un proceso intergubernamental abierto, inclusivo y participativo, conducente a la adopción de un nuevo conjunto de objetivos de alcance universal, que integren las tres dimensiones del desarrollo sostenible y sean aplicables a todos los países, desarrollados y en desarrollo: los ODS, llamados a constituirse en el elemento central de lo que entonces de denominó la agenda de desarrollo para después de 2015.

La propuesta original provino de Colombia, siendo el Perú el primero en apoyarla e integrar un grupo de países que condujo el esfuerzo diplomático necesario para alcanzar una masa crítica de apoyo a su inclusión en la declaración final de la Conferencia Rio+20, intitulada «El futuro que queremos». El Perú apoyó decididamente la propuesta, pues luego de haber alcanzado satisfactoriamente los ODM 
comprendió que el objetivo del desarrollo es una tarea inconclusa mientras los sectores más vulnerables queden fuera del progreso económico y social, o relegados por el sistema de medición del cumplimiento de las metas que privilegia los promedios nacionales. En esos años el Perú enfatizó la importancia de la inclusión social para erradicar la pobreza y fue cobrando una mayor conciencia sobre el potencial de su megadiversidad biológica y la necesidad de enfrentar su deterioro y las vulnerabilidades climáticas que el país enfrenta.

La elaboración de la Agenda 2030, incluyendo la Agenda de Addis Abeba sobre Financiación para el Desarrollo, tomó cerca de tres ańos. En la primera fase, dedicada a los ODS, se impuso una modalidad novedosa de negociación intergubernamental, sobre la base de un grupo de trabajo abierto a la participación de todos los Estados, la sociedad civil, el sector privado y la academia. El proceso contó también con la encuesta «El mundo que queremos», que consultó a más de un millón de personas en 88 países. Las modalidades de trabajo evitaron la tradicional polarización entre países desarrollados y en desarrollo, en la que los segundos negocian a través del Grupo de los 77 y China, y favorecieron un esquema más cooperativo que paradójicamente permitió un documento más ambicioso para los intereses del mundo en desarrollo.

Los 17 ODS y sus 169 metas son definidos como un conjunto integrado e indivisible, cuyo progreso será medido por el marco global de indicadores a ser monitoreado por las agencias de estadística de cada país en cooperación con la Comisión de Estadística de las Naciones Unidas. La Agenda 2030 aporta además nuevos paradigmas para la cooperación en el ámbito del desarrollo, desde una perspectiva de derechos centrada en la persona y en la dignidad humana. Está llamada a no dejar a nadie atrás y es aplicable a todos los países, y al mismo tiempo es respetuosa de las distintas circunstancias nacionales. En ese sentido, corresponde a cada país apropiarse de ella e implementarla de acuerdo con esas circunstancias y con las consecuentes necesidades y prioridades. La Agenda 2030 reconoce asimismo la diversidad cultural del mundo, y que la cultura es un catalizador clave del desarrollo sostenible.

La responsabilidad principal de su implementación corresponde a los estados en sus respectivos territorios. Sus esfuerzos, para estar a la altura de una agenda que aspira a ser transformadora, deben no obstante ser apoyados por una alianza mundial para el desarrollo sostenible en la que están llamados a participar el sector privado, la sociedad civil, la academia, las instituciones financieras internacionales, el sistema de las Naciones Unidas y, en general, todos los actores relevantes. Estos incluyen a todas las personas, que son reconocidas además como las destinatarias finales de todos los esfuerzos por erradicar la pobreza y alcanzar el desarrollo sostenible. 
La Agenda 2030 contempla también la necesidad de proveer medios de implementación para el logro de los ODS. Cada uno de estos incluye metas sobre dicha provisión, y el ODS 17 está dedicado enteramente al establecimiento de una alianza mundial para el desarrollo sostenible. Los medios de implementación son apoyados, complementados y contextualizados por las políticas y acciones incluidas en la Agenda de Acción de Addis Abeba sobre Financiación para el Desarrollo, que es parte integral de la Agenda 2030, propugna la movilización de recursos financieros de fuentes públicas y privadas, nacionales e internacionales y, entre otros medios de implementación, establece un mecanismo para la ciencia y la tecnología.

Las posiciones peruanas en el proceso de elaboración de la Agenda 2030 estuvieron sustentadas en los aportes de un mecanismo informal de coordinación establecido por la Cancillería con todos los sectores concernidos, así como con representantes de la sociedad civil. Gracias a ello, el Perú pudo participar activa y solventemente en las negociaciones, haciendo dupla con México, y en determinadas instancias en cuarteto, sumando a la dupla conformada por Colombia y Guatemala. En las fases finales otros países latinoamericanos como Costa Rica, Chile y Panamá, entre otros, fueron sumándose a las intervenciones generadas desde el referido cuarteto, siempre en consistencia con la visión del desarrollo y las prioridades identificadas en el Perú.

El Perú también presidió dos grupos informales, cada uno conformado por más de treinta países provenientes de todos los grupos regionales, abocados a la promoción, respectivamente, de la incorporación en la Agenda 2030 de metas asociadas a la cultura y a la inclusión financiera, y de su reconocimiento como catalizadores del desarrollo sostenible. El Perú integró asimismo un grupo informal promotor del desarrollo de la nińez y la juventud, así como el denominado Grupo de los 7, junto con Egipto, Liechtenstein, Noruega, Pakistán, República de Corea y Suiza, abocado a construir un consenso conducente al establecimiento de un marco de seguimiento y examen de la Agenda 2030, de carácter abierto e inclusivo en tres niveles de implementación: local, regional y global.

La coherencia y el liderazgo del Perú durante el proceso de elaboración de la Agenda 2030 fueron reconocidos durante la sesión final de negociación de los ODS, cuando se le encargó la responsabilidad de alcanzar un compromiso final sobre el Objetivo 16. Este fue sin duda el más controvertido durante todo el proceso, por incluir elementos como el acceso a la justicia, el Estado de derecho, la transparencia y la rendición de cuentas de instituciones inclusivas, que algunos países consideraban no correspondían al ámbito del desarrollo sostenible y podía conducir a la «securitización» de la Agenda 2030, invadiendo ámbitos reservados al Consejo de Seguridad. 
En alcance al llamado incluido en la Agenda 2030 para que cada región identifique un foro para dar seguimiento y examen a su implementación en ese ámbito, en ejercicio de la presidencia pro tempore de la Comisión Económica de las Naciones Unidas para América Latina y el Caribe (CEPAL), en 2016 el Perú también facilitó la creación del primer foro regional sobre desarrollo sostenible, cuya primera sesión se celebró con éxito en la Ciudad de México en abril de 2017.

La Agenda 2030 es altamente consistente con las posiciones llevadas por el Perú a las negociaciones, elaboradas sobre la base de su propia experiencia con los ODM y de las políticas de estado que impulsa. Así, la Agenda 2030 define a la pobreza como un fenómeno multidimensional cuya erradicación requiere atender sus causas estructurales, proteger a las personas y los segmentos más vulnerables de la población, reducir las desigualdades y luchar contra la discriminación. Las vulnerabilidades a ser atendidas incluyen expresamente las generadas por el cambio climático y por los desastres naturales.

El ODS 13 incorpora, como uno de los objetivos interconectados e indivisibles de la Agenda 2030, la necesidad de enfrentar el cambio climático, estableciendo expresamente un vínculo con la Convención Marco de las Naciones Unidas sobre el Cambio Climático. El Perú, país anfitrión de la Conferencia de las Partes de dicha Convención en 2014 - COP 20, junto con Francia, país anfitrión de la COP 21, jugó un papel clave en la promoción de dicho vínculo y de los consensos que finalmente permitieron adoptar el Acuerdo de París, tres meses después de la adopción de la Agenda 2030. El Llamado de Lima a la Acción Climática fue decisivo para generar el momento político requerido para alcanzar ese resultado.

La Agenda 2030 presta particular atención a la igualdad de oportunidades, incluyendo objetivos y metas sobre el acceso universal a servicios básicos entre los que se incluye la educación, la salud, la agricultura y la seguridad alimenticia, el agua y el saneamiento, así como sobre la igualdad de género y el empoderamiento de la mujer, sobre la promoción del crecimiento económico inclusivo, del empleo digno, de la infraestructura, de ciudades sostenibles, de sociedades pacíficas e inclusivas, y de instituciones transparentes y rendidoras de cuentas, además de objetivos sobre la conservación y uso sostenible de los ecosistemas terrestres y marinos, entre otros.

La consistencia de la Agenda 2030 con las necesidades y prioridades del Perú fue ratificada por el presidente Kuczynski en su discurso inaugural, y en consecuencia los ODS vienen siendo incorporados en el plan nacional de desarrollo por el Centro Nacional de Planeamiento Estratégico (CEPLAN), que es el punto focal peruano para el seguimiento correspondiente. El Instituto Nacional de Estadística e Informática (INEI), por su parte, tiene a su cargo el monitoreo de los indicadores correspon- 
dientes a las metas asociadas a los ODS, y ha publicado una base de datos en línea que puede ser consultada por todos los interesados ${ }^{3}$. El progreso peruano en la implementación de la Agenda 2030 será objeto de un primer examen nacional voluntario, a presentarse ante el Foro Político de Alto Nivel sobre Desarrollo Sostenible ${ }^{4}$, junto con otros 43 países, en julio de 2017 en las Naciones Unidas.

En línea con la prioridad asignada por su gobierno al acceso universal al agua y el saneamiento, en setiembre de 2016 el presidente Kuczynski fue invitado a incorporarse al Panel de Alto Nivel del Agua, creado por el secretario general de las Naciones Unidas y el presidente del Banco Mundial con el objetivo de movilizar actores y recursos para acelerar el logro del ODS 6, que precisamente busca garantizar la disponibilidad de agua y su gestión sostenible y el saneamiento para todos. El Perú también es un activo miembro del grupo de amigos de los océanos y ha apoyado decididamente la Conferencia sobre Océanos, a celebrarse en Nueva York del 5 al 9 de junio de 2017, con el objetivo de dar seguimiento a la implementación del ODS 14 sobre la preservación y el uso sostenible de los océanos y sus recursos. En concordancia con ello, el Perú ha copresidido en los dos últimos años el proceso oficioso de consultas cobre océanos y derecho del mar, impulsando una mayor convergencia de este con el desarrollo sostenible y el cambio climático.

Entre sus numerosas provisiones, cabe finalmente destacar que la Agenda 2030 reconoce expresamente que el desarrollo sostenible no puede hacerse realidad sin que haya paz y seguridad, y que la paz y la seguridad corren peligro sin el desarrollo sostenible.

\section{El secretario general António Guterres y el sostenimiento de la paz}

De cara al complejo contexto internacional observado en 2016, en particular por las diferencias entre los miembros permanentes del Consejo de Seguridad, para muchos resultó sorprendente que en octubre pasado la ONU pudiera elegir a su nuevo secretario general de manera consensual, sin mayores sobresaltos, y dentro de los plazos establecidos. Máxime cuando en este último proceso de selección, que tuvo mayor transparencia que los anteriores, se llegó a contar hasta doce candidatos, seis de ellos mujeres, todos con muy buenas credenciales, provenientes de hasta tres grupos regionales distintos.

Ver http://ods.inei.gob.pe/ods/inicio.html

El HLPF, por sus siglas en inglés, es el foro convocado anualmente a nivel ministerial por el ECOSOC y cuatrienalmente a nivel presidencial por la Asamblea General, para dar seguimiento y examinar la implementación de la Agenda 2030 en el ámbito global. 
De acuerdo con la Carta de las Naciones Unidas, el Consejo de Seguridad recomienda el nombre del candidato a la Asamblea General. En la práctica, históricamente, ello significó que la decisión tomada en el Consejo —en donde los cinco miembros permanentes tienen derecho de veto- dejaba a la Asamblea poco margen para influir en el proceso. En 2016, no obstante, por primera vez toda la membresía pudo conocer con anticipación a todos los candidatos, escuchar sus propuestas e interactuar con ellos. Los quince miembros del Consejo pudieron con ello ponderar las opiniones formadas en los demás miembros de la ONU, antes de adoptar su decisión.

Bajo esas modalidades, los antecedentes y las capacidades personales y profesionales de António Guterres, ex primer ministro de Portugal y ex alto comisionado de las Naciones Unidas para los Refugiados, lo posicionaron desde un inicio como uno de los más serios aspirantes al cargo. Guterres ocupó los primeros lugares en todas las seis votaciones simuladas que tuvieron lugar a puertas cerradas en el Consejo de Seguridad, hasta lograr la unanimidad. Su elección por la Asamblea General se produjo luego por aclamación, suscitando una gran expectativas.

La gestión del nuevo secretario general enfrenta, entre sus diversos desafíos, el de movilizar una renovada alianza global para implementar los instrumentos adoptados durante la gestión del surcoreano Ban Ki-moon (2008-2016), en particular la Agenda 2030 para el Desarrollo Sostenible y el Acuerdo de París, que representan una oportunidad concreta para transformar el mundo erradicando la pobreza y asegurando su sostenibilidad. En especial el segundo es considerado el legado principal de Ban Ki-moon, quien dedicó buena parte de su gestión a asegurar que las negociaciones sobre cambio climático llegaran a buen término y que no se repitiera la frustración de la COP 15, realizada en 2009 en Copenhague.

En concreto, el logro de los Objetivos de Desarrollo Sostenible, incluyendo la implementación del Acuerdo de París, aun cuando el compromiso de la nueva administración estadounidense con el segundo venga siendo objeto de una revisión, implican un horizonte y un mandato para Guterres. Esto contrasta con la situación heredada en el ámbito de la paz y la seguridad internacionales, donde se observa un escenario incierto, en el que resulta urgente contar con un marco claro de acción.

\footnotetext{
5 El avance en materia de transparencia y democratización del proceso para elegir al más alto funcionario de la organización, se debió en buena medida a las diversas gestiones llevadas a cabo por varios países miembros de la organización, en particular de aquellos que integran el denominado grupo ACT (Accountability, Coherence and Transparency), del cual el Perú forma parte. Igualmente, desde la sociedad civil hubo una gran presión a favor de la transferencia.
} 
Bank Ki-moon tuvo que hacer frente a un contexto particularmente complejo en este ámbito. Durante su gestión se agudizaron los conflictos en el medio oriente, luego de la denominada primavera árabe, así como en determinados países africanos, y se internacionalizaron grupos terroristas como el mal llamado Estado Islámico. Se hicieron patentes las limitaciones de la comunidad internacional para responder al desastre humanitario generado por el conflicto en Siria, a pesar de la recurrencia de crímenes atroces y del altísimo número de víctimas fatales, desplazados y refugiados. De otro lado, se observó un deterioro en las relaciones entre las potencias occidentales y la Federación Rusa en torno a la situación en Ucrania, que repercutió negativamente en la confianza requerida entre dichas potencias para trabajar juntas a favor de la paz y la seguridad internacionales a través del Consejo de Seguridad.

El amplio respaldo con el que el nuevo secretario general inicia su gestión está relacionado en gran medida con su capacidad para articular una nueva visión política para enfrentar en forma más oportuna y eficaz los desafíos que actualmente enfrenta la ONU. Esta visión destaca la necesidad de implementar en forma coherente e integrada los importantes instrumentos adoptados por la comunidad internacional en 2015 en el ámbito del desarrollo sostenible, el cambio climático, la financiación del desarrollo y la prevención del riesgo de desastres. Pero, en adición a ellos, haciendo suyos los planteamientos sobre el sostenimiento de la paz incluidos en las resoluciones idénticas adoptadas en abril de 2016 por la Asamblea General y el Consejo de Seguridad ${ }^{6}$, Guterres propone el resurgimiento de una «diplomacia para la paz» que no solo haga frente a los actuales conflictos y crisis humanitarias, sino que se asegure que no surjan nuevos escenarios similares, poniendo especial énfasis en la prevención.

$\mathrm{Al}$ efecto, el secretario general considera que el logro de los ODS y el sostenimiento de la paz implican agendas complementarias que se refuerzan mutuamente, en la medida que el desarrollo sostenible cimienta la paz y esta a su vez habilita el desarrollo. Su aproximación a estos desafíos globales enfatiza la necesidad de estrechar la colaboración entre los tres pilares de la ONU: paz y seguridad internacionales, desarrollo y derechos humanos. Ello con miras a atender las causas profundas de los conflictos. Propone en ese sentido una arquitectura moderna, efectiva y operacional para el sostenimiento de la paz, capaz de contribuir a ella antes, durante y después del estallido de un conflicto. En esta visión, la prevención es identificada como una orientación práctica, que implica, entre otros alcances, el despliegue de todos los esfuerzos posibles para ayudar a los países a evitar el estallido de crisis, el uso y el fortalecimiento de las capacidades de alerta temprana para la oportuna interposi-

6 Resoluciones 70/262 de la Asamblea General y 2282 (2016) del Consejo de Seguridad, respectivamente. 
ción de buenos oficios, la mediación, y la integración de la perspectiva de género en dichos esfuerzos?

Cabe referir que las mencionadas resoluciones idénticas sobre el sostenimiento de la paz siguieron en gran medida las recomendaciones de un grupo de expertos convocado por el entonces secretario general Ban Ki-moon para analizar la arquitectura del sistema de las Naciones Unidas para la consolidación de la paz, que en junio de 2015 produjo un informe intitulado "El desafío del sostenimiento de la paz»" Dicho grupo estuvo presidido por el destacado diplomático y académico latinoamericano Gert Rosenthal, quien como representante permanente de Guatemala ante las Naciones Unidas cumplió una reconocida labor como miembro no permanente del Consejo de Seguridad durante el período 2012-2013.

La necesidad de actuar preventivamente no es sin embargo un planteamiento nuevo. La propia Carta de las Naciones Unidas señala, en su artículo primero, que uno de los propósitos de la organización es el de mantener la paz y la seguridad internacionales, y con tal fin tomar, entre otras, «medidas colectivas eficaces para prevenir y eliminar amenazas a la paz». Asimismo, el artículo 34 establece que el Consejo de Seguridad "podrá investigar toda controversia, o toda situación susceptible de conducir a fricción internacional o dar origen a una controversia, a fin de determinar si la prolongación de tal controversia o situación pueda poner en peligro el mantenimiento de la paz y seguridad internacionales».

El mandato de la Carta es claro, y los desastres humanitarios de la década de 1990, en especial los genocidios y crímenes contra la humanidad ocurridos en Rwanda y la ex Yugoslavia, motivaron diversos informes del secretario general y resoluciones del Consejo de Seguridad que ponían el énfasis en medidas de prevención y alerta temprana9. En la práctica, no obstante, en muchos casos la aplicación de acciones de prevención ha enfrentado a gobiernos opuestos a que se investiguen situaciones en sus países o en países aliados, invocando al efecto los principios de soberanía y no intervención en los asuntos internos igualmente consagrados en la Carta.

El rechazo de la opinión pública a la recurrencia de crímenes atroces favoreció que, en respuesta a ello, la Declaración de Cumbre de las Naciones Unidas de $2005^{10}$

\footnotetext{
7 Carta del Secretario General del 3 de mayo de 2017, por la que informa a los Estados miembros de la ONU sobre las iniciativas y los procesos de reforma que su administración viene impulsando (ONU, 2017).

8 Circulado entre los Estados miembros con carta del 29 de junio de 2015.

9 En el ańo 2000 se publicó en Naciones Unidas un informe sobre las operaciones de paz, elaborado por un panel de expertos y conocido como el Informe Brahimi, en el que se dieron diversas recomendaciones sobre cómo mejorar estas operaciones que enfatizaron la importancia de la prevención. Y en 2001 el Secretario General publicó un informe específicamente sobre «Prevención de los Conflictos Armados».

10 Adoptada mediante resolución 60/1 de la Asamblea General. Ver párrafos 58 y 59.
} 
incluyera el concepto de la «responsabilidad de proteger»(R2P), según el cual la prevención de crímenes atroces exige que se asignen responsabilidades a los Estados y a la comunidad internacional y se promueva la colaboración entre ellos. Así, la obligación de prevenir y detener el genocidio y los crímenes de guerra, la depuración étnica y los crímenes de lesa humanidad, así como de la incitación a ellos, incumbe ante todo al Estado involucrado, pero compete a la comunidad internacional desempeñar un papel que no puede verse coartado en nombre de la soberanía. Con ello la soberanía no significa únicamente protección de los Estados frente a injerencias extranjeras, sino que constituye una carga de responsabilidad que los obliga a prevenir y actuar para garantizar el bienestar de su población.

El concepto se llevó a la práctica en algunos casos pero no ha dejado de generar controversia, principalmente luego de que se invocara en la resolución 1973 (2011) por la que el Consejo de Seguridad autorizó la intervención en Libia con el objeto de proteger a la población civil, desencadenando lo que a la postre fue la caída del régimen de Muammar Gadafi y una guerra civil en ese país ${ }^{11}$. Ulteriores iniciativas en torno al concepto R2P no han llevado a respuestas consistentes por parte del sistema de Naciones Unidas, como resulta evidente en particular respecto del caso de Siria. Frente a la frustración generada por tales antecedentes, el nuevo secretario general plantea una nueva esperanza para la prevención, a partir de una clara decisión, sustentada en acciones concretas, de hacer de la prevención y de la acción coherente y coordinada del sistema, el eje de su gestión para lograr tanto el sostenimiento de la paz como el desarrollo sostenible.

En la visión de Guterres sobre el sostenimiento de la paz, el énfasis en la prevención tiene como correlato una agenda aspiracional y holística, como es la del desarrollo sostenible. La designación de Amina Mohammed como subsecretaria general, sin duda da cuenta del compromiso de Guterres con el empoderamiento de la mujer y con la prioridad asignada al África, pero sobre todo con la implementación de la Agenda 2030 y con la reforma del sistema de las Naciones Unidas para lograr que su apoyo a los países miembros sea más coherente e integrada, y signifique en el terreno una acción más coordinada de sus tres pilares. La señora Mohammed, exministra del Ambiente de Nigeria, goza de un alto prestigio por el papel que cumplió durante la negociación de la Agenda 2030 como representante especial del entonces secretario general Ban Ki-moon. Se le ha encargado precisamente conducir un equipo de trabajo responsable de implementar la última revisión cuatrienal de las políticas que orientan al sistema de las Naciones Unidas para el desarrollo, acordada por la Asamblea General en diciembre de 2016.

\footnotetext{
11 Las principales críticas y resistencias al concepto R2P argumentan su utilización por las potencias occidentales para intervenir en otros países y cambiar regímenes de manera selectiva.
} 
La embajadora brasilera Maria Luiza Ribeiro Viotti, desde su posición como jefa de Gabinete del Secretario General, por su parte preside un comité ejecutivo que se reúne semanalmente con el objetivo de promover una mayor integración entre los tres pilares de la Organización. Por directivas del secretario general, las importantes Direcciones de Asuntos Políticos y de Operaciones de Mantenimiento de la Paz, vienen trabajando de manera más estrecha y coordinada, promoviendo sinergias entre ellas. Se ha creado asimismo una comisión encargada de hacer un levantamiento de todas las herramientas de prevención con que cuenta el sistema de las Naciones Unidas para hacerlo más eficiente.

Entre sus primeras medidas, António Guterres ha decidido asimismo crear una oficina, a ser encabezada por un secretario general alterno, para centralizar todos los programas que Naciones Unidas impulsa en materia de lucha contra el terrorismo, reconociendo la gravedad de este flagelo y la necesidad de una respuesta integrada en línea con el énfasis puesto por su gestión en el sostenimiento de la paz. Esto al tiempo de elevar el perfil de la ONU en un asunto particularmente delicado en el actual contexto internacional.

Con estas acciones, el secretario general se posiciona políticamente en un contexto que se presenta especialmente incierto para el multilateralismo y la ONU por la emergencia de nacionalismos que vienen cuestionando la vigencia del orden internacional, lo que a su vez supone serias implicancias para el comercio y la cooperación internacional requerida para enfrentar desafíos globales como la pobreza y el cambio climático. En atención a ese escenario, en un reciente artículo de opinión, el canciller del Perú, Ricardo Luna (2017), se refería a la emergencia de una crisis sistémica global sin paralelos desde el período entre las dos guerras mundiales, señalando que «en este tumultuoso e impredecible mundo es necesario que el Perú contribuya a la reingeniería y al afianzamiento de las instituciones multilaterales», y reconociendo que «el liderazgo del nuevo Secretario General de las Naciones Unidas, António Guterres, es un punto de partida y un claro ejemplo de una visión coincidente con la política exterior de profundas raíces éticas que siempre ha inspirado a nuestro país». Estas reflexiones sobre los desafíos de la política exterior del Perú, y la voluntad de seguir contribuyendo al multilateralismo y a un orden mundial basado en reglas internacionales, tienen especial significación por cuanto van acompañadas de una evaluación descarnada de la complejidad de los desafíos del actual contexto internacional. Esta es coincidente, por ejemplo, con la expresada en un reciente artículo por el presidente del Grupo Internacional sobre Crisis, el diplomático y académico francés Jean Marie Guéhenno: «Los pesimistas tenían razón, por lo menos en el corto plazo las cosas se están poniendo peor. El terrorismo y los conflictos armados se han 
incrementado en la década pasada, y la visión post Segunda Guerra Mundial de un orden mundial cooperativo, que parecía tener una segunda oportunidad con el fin de la Guerra Fría, se encuentra actualmente amenazado por el resurgimiento del nacionalismo" (2017).

En este orden de ideas es que la temprana visita del presidente Kuczynski al nuevo secretario ejecutivo, y su oportuno mensaje de apoyo al multilateralismo, revisten una particular relevancia. Máxime cuando el Perú se prepara para ocupar un lugar no permanente en el Consejo de Seguridad durante el período 2018-2019. Esto de conformidad con el artículo 23 de la Carta de las Naciones Unidas, el cual prevé que, además de los cinco miembros permanentes, se elegirá a diez miembros no permanentes "prestando especial atención, en primer término, a la contribución de los Miembros de las Naciones Unidas al mantenimiento de la paz y seguridad internacionales y demás propósitos de la Organización».

El Perú, como parte de la campaña para su elección, ha resaltado su vocación por la paz y su tradición de servicio a la comunidad internacional, manifestada, entre otras contribuciones, en acciones concretas como la participación en operaciones de mantenimiento de la paz. Estas constituyen el principal instrumento con el que cuenta el Consejo de Seguridad para desarrollar su labor, y han crecido en número y en tamaño en los últimos años, debido, entre otros factores, a la recurrencia de graves conflictos políticos internos, al terrorismo y a la violencia interétnica que se han ido produciendo en diversos países, principalmente del continente africano. Se viene llevando a cabo un proceso de revisión del funcionamiento de estas operaciones a fin de adecuarlas a las nuevas realidades. El Perú ha contribuido con más de ocho mil efectivos en diversas misiones a lo largo de los últimos cincuenta ańos, siendo de destacar su participación en la Misión de Estabilización de Naciones Unidas en Haití (MINUSTAH), y más recientemente en la República Centroafricana, con una compañía de ingeniería.

En adición a ello, siendo un país consciente de las potenciales crisis humanitarias y los conflictos que para la humanidad representan las vulnerabilidades al cambio climático, los desastres naturales, la escasez de agua, o el extremismo violento, el Perú espera contribuir al establecimiento de una oportuna coordinación por parte del sistema de las Naciones Unidas, que asegure adecuados flujos de información para contar con eficaces sistemas de alerta temprana con los cuales fortalecer las capacidades del Consejo de Seguridad para la prevención y la gestión de conflictos y crisis. Cabe recordar que el actual conflicto en Siria se inició por las protestas suscitadas por una sequía generada a su vez por el cambio climático. Tal es la complejidad de los desafíos a los que se enfrenta el Consejo de Seguridad. 


\section{Conclusión}

La diplomacia peruana se prepara para cumplir el mismo papel constructivo e innovador, al servicio de la comunidad internacional, que caracterizó su participación en la negociación de la Agenda 2030 para el Desarrollo Sostenible y que refleja su tradición de apoyo al multilateralismo. País comprometido con la defensa y promoción del derecho internacional y de los principios y propósitos consagrados en la Carta de Naciones Unidas, y consciente de la existencia de nuevos desafíos a la paz y la seguridad internacionales, el Perú promoverá una acción más efectiva de este, en línea con su función de identificar nuevas amenazas y con el énfasis planteado por el secretario general en la prevención y la acción coherente e integrada requerida para el sostenimiento de la paz.

\section{Referencias}

Guéhenno, J-M. (2017, 29 marzo). War Must Not Become the New Normal. Journal of International Affairs, marzo. Recuperado de https://jia.sipa.columbia.edu/ war-must-not-become-new-normal

Luna, R. (2017). Desafios de la politica exterior peruana. El Comercio, edición del 29 de enero de 2017.

ONU (2017, 3 de mayo). Carta del Secretario General por la que informa a los Estados miembros de la ONU sobre las iniciativas y los procesos de reforma que su administración viene impulsando. Nueva York: Secretaría General de la ONU.

The Guardian (2012, 16 de noviembre). Mark Malloch-Brown: developing the MDGs was a bit like nuclear fusion. Recuperado de https://www.theguardian.com/ global-development/2012/nov/16/mark-malloch-brown-mdgs-nuclear

Fecha de recepción: 26 de mayo de 2017 Fecha de aprobación: 27 de junio de 2017 